\title{
Biological Disruptive Therapies as Counter Measures for Unpredictable Biological Insults, a Case for Successful Treatment of COVID-19
}

\author{
Mohammad Nezami1, Vicky Yamamoto² \\ ${ }^{1}$ Sahel Oncology LLC, Cancer Research and Life Foundation, Research Cancer Institutes of America, Newport Beach, CA, USA \\ ${ }^{2}$ Department of Head and Neck Surgery, School of Medicine, University of Southern California (USC), Los Angeles, CA, USA \\ Email: amnezami@yahoo.com
}

How to cite this paper: Nezami, M. and Yamamoto, V. (2020) Biological Disruptive Therapies as Counter Measures for Unpredictable Biological Insults, a Case for Successful Treatment of COVID-19. Advances in Infectious Diseases, 10, 175-183. https://doi.org/10.4236/aid.2020.103018

Received: August 8, 2020

Accepted: September 11, 2020

Published: September 14, 2020

Copyright $\odot 2020$ by author(s) and Scientific Research Publishing Inc. This work is licensed under the Creative Commons Attribution International License (CC BY 4.0).

http://creativecommons.org/licenses/by/4.0/

\begin{abstract}
Treatment of patients with severe COVID-19, is challenging specifically when a patient carries high risk of mortality, such as old age, immune suppression or cancer. Also a patient who manifests the disease with severe symptoms, such as hypoxia, requiring supplemental oxygenation, or artificial ventilation has a poor prognosis. Here we review the scientific rationale used to design a very promising therapy based on existing literature, but in significantly different method and protocols, used to treat cases of severe COVID-19, and we conclude that although the effort on drug development has been enormous, but as of today, we do not have a therapy with specific characteristics as this protocol, to be used safely in human and yet potentially meet the expectations we would have for a so called "effective therapy". Further clinical trials are needed to support this hypothesis and generate further hypothesis to prove the concept in larger cohort of patients.
\end{abstract}

\section{Keywords}

COVID-19, Acute Respiratory Distress Syndrome(ARDS), Phosphoinositol 3 Kinase (Pi3K), Quercetin

\section{Background}

Severe COVID-19 is a deadly disease in patients with cancer, or the patients who are on steroids or other immune suppressing conditions. The data have shown inferior results in outcome in these groups. Men also have higher risk of significant comorbidity and death. Among them, aged 75 and more are specifically at 
risk for death.

Studies in China showed COVID-19 patients with cancer had higher risks in all severe outcomes. Patients with hematological cancer, lung cancer, or with metastatic cancer (stage IV) had the highest frequency of severe events. Non-metastatic cancer patients experienced similar frequencies of severe conditions to those observed in patients without cancer. Patients who received surgery had higher risks of having severe events, while patients with only radiotherapy did not demonstrate significant differences in severe events when compared to patients without cancer. These findings indicate that cancer patients appear more vulnerable to SARSCOV-2 outbreak. Since this was the first large cohort study on this topic, the report will provide the much-needed information that will benefit global cancer patients. As such, the study was suggested to be disseminated widely to alert clinicians and patients [1]. Further initial results of the registry study of the COVID-19 and cancer consortium (CCC19; LBA11) in March 2020, with the objective to rapidly collect and disseminate prospective uniform data from patients with cancer who had been diagnosed with COVID-19, shows that in over 1035 cases, followed for 21 days, patients with cancer had a greater mortality of 13 percent compared with global mortlity rate of 6 percent and experienced higher rates of complications, including ARDS [2]. Further studies in Wuhan, investigated the epidemiological variations of the affected individuals with severe disease [3].

Application of a variety from natural products had been proposed and tested in the laboratory to treat Corona Virus infection since 1970s. One area of focus was the enterance of the virus [4] [5] and mechanistics involved which were studied in HIV and Herpes Virus [6] [7] [8] [9]. Many studies have investigated the role of inflammation and production of lactate in the COVID-19 pneumonia and ARDS [10] [11] [12] [13].

The question of quantity of human response to any of the proposed therapies, however seemed always to be a challenging question as the magnitude of response was always suboptimal in cases with severe disease, such as pneumonia or acute respiratory distress syndrome (ARDS), a severe inflammatory response in Cov19, manifested by lactate production secondary to activation of hypoxic cascade [14]-[20].

Recently there has been a significant effort in screening Chinese herbs and compounds to find a treatment which could disrupt the virus replication by inhibition of specific enzymes, dictated by specific genes [21]. These efforts yielded scientists in the field to believe that there is a potential therapy that could be used with no sign of resistance, at a dose dependent level, and yet has potentials for inhibition of selective Phosphoinositol kinase (Pi3K). This is a unique enzyme involved in growth of many solid tumor types, including breast cancer. There was enough clinical experience in our clinic, to use this compound in the form of intravenous application, to understand the exact clinical response in cancer patients, but no patient with COVID-19 was treated. 


\section{Methods and Results}

Quercetin was identified as the most potent inhibitor of the screened molecules to inhibit Pi3K mutated gene, based on laboratory findings [21] [22] [23] and was administered per (multi targeted epigenetic therapy) MTET protocol.

To date, 52 patients with activating mutations identified in their liquid biopsy test by Guardant 360 , were treated with this protocol, with serial monitoring of the response, through 250 test samples. These cases were tracked and their mutated allele frequencies measured longitudinally to document response and correlate with the clinical outcome. There was 89 percent response post treatment. Patients aged from 24 to 80 and the male/female ratio was 0.7 . Duration of therapy was between 2 weeks to 5 years, with minimum of ten treatments between the test/re test.

Here we present samples as cases to this article:

Case 1:

70 years old male with history of prostate cancer Gleason score of 9, diagnosed in April 2017, status post radiation as well as hormonal blockade with Lupron and Zytiga, so far he has received 2 shots of Lupron, at 3 months interval, PSA has decreased from 19 to 5. Patient has some urinary hesitation after the radiation, as well as severe fatigue.

Upon his arrival he was evaluated for metastatic disease with a whole body PET scan, which showed extensive bony mets, all through his pelvis, and vertebras.

His liquid biopsy on 10/24/17, showed positive c DNA for TP53, BRCA, PDGF, and APC. He was immediately started on IV epigenetic therapies which he received on daily basis, for two weeks, his labs were further repeated. His Prostate acid phosphatase dropped from 71 to 66. His PSA dropped from 11 to 7. During this time, he had stopped his Zytiga, and did not receive any conventional therapies. His quality of life improved and he no longer had fatigue. His bone scan was repeated on $12 / 2 / 17$, which showed stable disease. His labs show on $1 / 23 / 18$, that his TGF had dropped from 5207 down to 3372, and his PAP dropped from 71.6 to 70.8 (stable).

He further was started on Gleevec along with epigenetic therapies, and continued to respond clinically to the therapy. Noticeably he did not receive any hormonal blockade.

His PSA was checked on $2 / 14 / 18$ and it was down to 3.3 from 7.2 (measured on $2 / 14 / 18$ and $12 / 7 / 17$ respectively. He was restaged with a whole body PET scan on $3 / 13 / 18$ which showed sclerosis and healing of all his bony lesions as well as resolution of his paraaortic LN.

His CTC was repeated on 5/22/20 and it showed drastic reduction of ERBB2 and telomerases. (see below). His PSA also dropped from.9 in may 2020, to 6.8 on $6 / 22 / 20$, after the course of treatments.

On 5/17/2020 he randomly was tested for COVID-19, due to his exposure in NY. He was tested positive for COVID-19 antibody test (Both IgG and IgM)but negative for the antigen test (PCR) performed on 5/20/2020, and yet did not have any symptoms, as he was treated for his prostate cancer with same treat- 
ment. Surprisingly the wife who also was treated at the clinic for other conditions, never had a positive test, although being intimate with him. The risk of exposure was recognized as a trip to NY while the pandemic was identified at the NY State (Figure 1).

Case 2:

The case report related to this article has the most criteria for the worst outcome, expected from this disease. The case is a 77 year old male with history of mantle cell lymphoma treated for over 10 years by our clinic. He was initially diagnosed on 10/25/2011 with an orbital mass, confirmed by pathology status post Maxi-ChopX 4 cycles, protocol referred to us for metabolic and epigenetic evaluation and treatments. He refused further chemotherapy secondary to severe side effects. His labs were drawn on 1/30/2012 which revealed increased LDH. His pathology originally was positive for both bcl and Ki 67 and his macroglobulin was elevated, all suggestive of poor prognosis and aggressiveness of his cancer.

His initial labs showed LDH of 252, and ferritin of 850 and presence of metamyelocytes and myelocytes. Immediately he was started on the epigenetic and antioxidant IV protocol. After three sessions his fatigue improved and his function

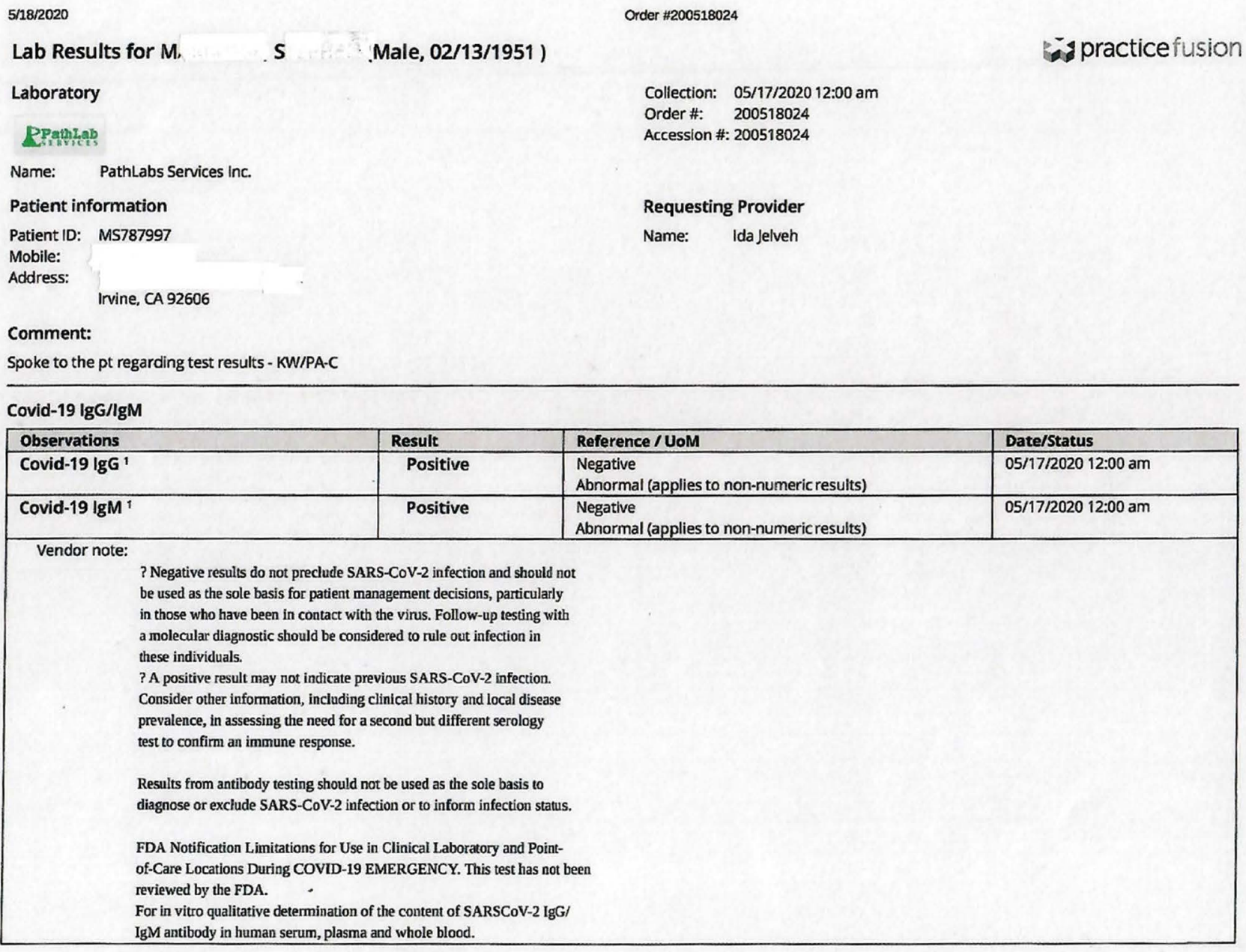


Patient information

Patient ID: MS787997

Mobile: $\quad$ 949-394-2427

Address: 16552 VON KARMAN AVENUE

Irvine, CA 92606
Requesting Provider

Name: Bryan Doonan

\section{Comment:}

$5 / 21$ notified

\section{SARS-CoV2 RT-PCR}

\begin{tabular}{|l|l|l|l|}
\hline Observations & Result & Reference / UoM & Date/Status \\
\hline SARS-CoV2 RT-PCR 1 & Negative & & $05 / 20 / 202011: 00$ am \\
\hline
\end{tabular}

https:/static.practicefusion.com/apps/ehr/index.html\#/PF/charts/patients/2ddaa5dc-7201-4e46-9a0c-cfc52eaa0922/results/dc6e7eee-c9ef-4212-8525-... 1/3

Figure 1. Patient, case 1, blood test results for COVID-19 antibodies.

increased. After 5 treatments, his labs were repeated. His LDH (tumor marker) dropped to 231, his ferritin to 509 and his beta 2 microglobulin showed normal results, when increased levels were seen in his electrophoresis before starting the treatments. 
His Meta myelocytes and myelocytes both disappeared in his labs after the 5 sessions of therapy. His LDH normalized at 132 on 3/13/2012. His CRP dropped from 56 to 0.5 between $2 / 6 / 2012$ and 4/2/2012. His natural killer cell activity increased from 14 to 19 between $2 / 1 / 2012$ to $5 / 3 / 2012$ and to 51.1 on $8 / 23 / 2012$. His IGF-1 dropped from 218 on 4/12/2012 to 133 on 5/3/2012.

His bone marrow biopsy was negative for malignancy on $3 / 20 / 2012$, and his PET scan was negative reported in April 2012, giving him diagnosis of complete clinical remission.

He had a local recurrence on his left orbit in November 2013, which was reported with a focal FDG avid lesion in PET scan. He immediately started the IV epigenetic therapy on twice weekly program with us and continued the program for 4 weeks, along with daily intake of Ibrutinib at $140 \mathrm{mg}$ per day. The restaging PET scan on 1/29/14, revealed complete resolution of the lesion with normal activity.

Further in 2015 he was diagnosed with Parkinson disease and treated accordingly. He also had increased PSA between 2017 and 2020 which was treated with active surveillance and IV epigenetic therapies.

On $6 / 22,23 / 2020$, he referred to the clinic for maintenance therapies for his lymphoma when he was suspected to have a recurrent disease in his PET scan at same site (left orbit). He responded well to the therapy with improved vision in three days, however on 7/1/2020, he developed cough and fever, severe fatigue and shortness of breath which required oxygen supplementation, as he developed hypoxia with pulse oximetry of 85 percent on room air. His physical examination was suggestive of pneumonia, with right lung field ronchi and crackes. He was tested positive for COVID-19 through PCR (nasal swab).

Immediately he was started on IV Hydrogen peroxide, Quercetin/PEG and EGCG which he received per protocol to treat COVID-19 on daily basis. His oxygenation improved over three days and his saturation increased from 85 percent on room air to 95 percent. His requirement for supplemental oxygen was lowered each and every time he received the Iv therapy. For example, on days 3 - 5 he was on 4 liters of oxygen, and on day 5 - 7, reduced to 2 liters only at night time.

He did not receive any other therapies during this time. His cough stopped in 5 days and his physical examination showed resolution of right pulmonary crackles. He was able to taper the therapy down from four times a week after three weeks, and further stopped on 7/22/20. He tested negative for COVID-19 PCR, on 7/24/2020 (See Figure 2).

\section{Conclusion}

This case report although small is sample size has significant relevance and statistical effect size. We certainly understand the limitations of the study including the lack of randomization or placebo control, but we also recognize the lack of effective therapies in this scenario, making it difficult to ethically treat a patient 


\section{green}

2021 Herndon Ave Suite 201 Clovis, CA 93611

559-813-

$\begin{array}{llll}\text { Patient Name: } & \text { Ma } & \text { DOB: } 06 / 04 / 19\end{array}$

Manny Fagundes was swabbed on 7/24/20 for a Rapid COVID 19

Nasopharyngeal Swab was done by FIA SARS Antigen testing.

The results of this testing were

Negative. If you have symptoms, please stay out of work for 3 days past symptom resolution. If you have no symptoms you can return to full duty immediately.

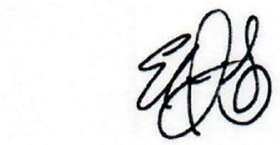

Erick J.

MD

Date $7 / 24 / 20$

If you have any questions, please feel free to call $559-813$

Figure 2. Patient (case 2), COVID-19 antigen test results.

with COVID ARDS with placebo. We observed unexpected results with positive clinical outcome in most difficult to treat patient with high risk of mortality. We recommend further studies to prove the concept on application of this protocol on larger groups of patients as critically ill to supplement the conclusion.

\section{Conflicts of Interest}

The author declares no conflicts of interest regarding the publication of this paper.

\section{References}

[1] Dai, M.Y., et al. (2020) Patients with Cancer Appear More Vulnerable to SARSCoV-2: A Multi-Center Study during the COVID-19 Outbreak. Cancer Discovery, 10, 783-791.

[2] Stenger, M. (2020) Results of a COVID-19 and Cancer Consortium (CCC19) Cohort Study-The ASCO Post.

[3] Chen, N.S., Zhou, M., Dong, X., Qu, J.M., Gong, F.Y., Han, Y., et al. (2020) Epidemiological and Clinical Characteristics of 99 Cases of 2019 Novel Coronavirus Pneu- 
monia in Wuhan, China: A Descriptive Study. The Lancet, 395, 507-513. https://doi.org/10.1016/S0140-6736(20)30211-7

[4] Bultmann, H. and Brandt, C.R. (2002) Peptides Containing Membrane-Transiting Motifs Inhibit Virus Entry. The Journal of Biological Chemistry, 277, 36018-36023. https://doi.org/10.1074/jbc.M204849200

[5] Bultmann, H., Busse, J.S. and Brandt, C.R. (2001) Modified FGF4 Signal Peptide Inhibits Entry of Herpes Simplex Virus Type 1. Journal of Virology, 75, 2634-2645. https://doi.org/10.1128/JVI.75.6.2634-2645.2001

[6] Dragic, T., Trkola, A., Thompson, D.A., Cormier, E.G., Kajumo, F.A., Maxwell, E., Lin, S.W., Ying, W., Smith, S.O., Sakmar, T.P. and Moore, J.P. (2000) A Binding Pocket for a Small Molecule Inhibitor of HIV-1 Entry within the Transmembrane Helices of CCR5. Proceedings of the National Academy of Sciences of the United States of America, 97, 5639-5644. https://doi.org/10.1073/pnas.090576697

[7] Este, J.A. (2003) Virus Entry as a Target for Anti-HIV Intervention. Current Medicinal Chemistry, 10, 1617-1632. https://doi.org/10.2174/0929867033457098

[8] Agelidis, A.M. and Shukla, D. (2015) Cell Entry Mechanisms of HSV: What We Have Learned in Recent Years. Future Virology, 10, 1145-1154.

https://doi.org/10.2217/fvl.15.85

[9] Guan, Y., Zheng, B.J., He, Y.Q., Liu, X.L., Zhuang, Z.X., Cheung, C.L., Luo, S.W., Li, P.H., Zhang, L.J., Guan, Y.J., Butt, K.M., Wong, K.W., Chan, K.L., Lim, W., Shortridge, K.F., Yuen, K.Y., Peiris, J.S. and Poon, L.L. (2003) Isolation and Characterization of Viruses Related to the SARS Coronavirus from Animals in Southern China. Science, 302, 276-278. https://doi.org/10.1126/science.1087139

[10] Drosten, C., Gunther, S., Preiser, W., Van der Werf, S., Brodt, H.R., Becker, S., Rabenau, H., Panning, M., Kolesnikova, L., Fouchier, R.A., Berger, A., Burguiere, A.M., Cinatl, J., Eickmann, M., Escriou, N., Grywna, K., Kramme, S., Manuguerra, J.C., Muller, S., Rickerts, V., Sturmer, M., Vieth, H., Klenk, D.S., Osterhaus, A.D., Schmitz, H. and Doerr, H.W. (2003) Identification of a Novel Coronavirus in Patients with Severe Acute Respiratory Syndrome. The New England Journal of Medicine, 348, 1967-1976. https://doi.org/10.1056/NEJMoa030747

[11] Kindler, E. and Thiel, V. (2016) SARS-CoV and IFN: Too Little, Too Late. Cell Host Microbe, 19, 139-141. https://doi.org/10.1016/j.chom.2016.01.012

[12] Yoshikawa, T., Hill, T., Li, K., Peters, C.J. and Tseng, C.T.K. (2009) Severe Acute Respiratory Syndrome (SARS) Coronavirus-Induced Lung Epithelial Cytokines Exacerbate SARS Pathogenesis by modulating Intrinsic Functions of Monocyte-Derived Macrophages and Dendritic Cells. Journal of Virology, 83, 3039-3048. https://doi.org/10.1128/JVI.01792-08

[13] Channappanavar, R., Fehr, A.R., Vijay, R., Mack, M., Zhao, J., Meyerholz, D.K. and Perlman, S. (2016) Dysregulated Type I Interferon and Inflammatory Monocytemacrophage Responses Cause Lethal Pneumonia in SARS-CoV-Infected Mice. Cell Host Microbe, 19, 181-193. https://doi.org/10.1016/j.chom.2016.01.007

[14] Zhang, D., Tang, Z.Y., Huang, H., Zhou, G.L., Cui, C., Weng, Y.J., et al. (2019) Metabolic Regulation of Gene Expression by Histone Lactylation. Nature, 574, 475-580. https://doi.org/10.1038/s41586-019-1678-1

[15] O’Neill, L.A., Kishton, R.J. and Rathmell, J. (2016) A Guide to Immunometabolism for Immunologists. Nature Reviews Immunology, 16, 553-565. https://doi.org/10.1038/nri.2016.70

[16] Tannahill, G.M., Curtis, A.M., Adamik, J., Palsson-McDermott, E.M., McGettrick, A.F., Goel, G., et al. (2013) Succinate Is an Inflammatory Signal That Induces IL-1 $\beta$ 
through HIF-1 $\alpha$. Nature, 496, 238-242. https://doi.org/10.1038/nature11986

[17] Clement, C., Tiwari, V., Scanlan, P.M., Valyi-Nagy, T., Yue, B.Y. and Shukla, D. (2006) A Novel Role for Phagocytosis-Like Uptake in Herpes Simplex Virus Entry. Journal of Cell Biology, 174, 1009-1021. https://doi.org/10.1083/jcb.200509155

[18] Macintyre, A.N., Gerriets, V.A., Nichols, A.G., Michalek, R.D., Rudolph, M.C., Deoliveira, D., et al. (2014) The Glucose Transporter Glut1 is Selectively Essential for CD4 T Cell Activation and Effector Function. Cell Metabolism, 20, 61-72. https://doi.org/10.1016/j.cmet.2014.05.004

[19] Zheng, W., Wang, G., Xu, Z-G., et al. (2019) Lactate Is a Natural Suppressor of RLR Signaling by Targeting MAVS. Cell, 178, 176-189.

https://doi.org/10.1016/j.cell.2019.05.003

[20] Lottes, R.G., Newton, D.A., Syropoulos, D.D. and Baetz, J.E. (2015) Lactate as Substrate for Mitochondrial Respiration in Alveolar Epithelial Type II Cells. The American Journal of Physiology-Lung Cellular and Molecular Physiology, 309, L953-L961. https://doi.org/10.1152/ajplung.00335.2014

[21] Xie, J.C., Zhu, L.L., Luo, H.P., Zhou, L., Li, C.X. and Xu, X.J. (2001) Direct Extraction of Specific Pharmacophoric Flavonoids from Gingko Leaves Using a Molecularly Imprinted Polymer for Quercetin. Journal of Chromatography A, 934, 1-11. https://doi.org/10.1016/S0021-9673(01)01294-8

[22] Wen, C.C., et al. (2007) Specific Plant Terpenoids and Lignoids Possess Potent Antiviral Activities Against Severe Acute Respiratory Syndrome Coronavirus. Journal of Medicinal Chemistry, 50, 4087-4095. https://doi.org/10.1021/jm070295s

[23] Ling, Y., et al. (2004) Small Molecules Blocking the Entry of Severe Acute Respiratory Syndrome Coronavirus into Host Cells. Journal of Virology, 78, 11334-11339. https://doi.org/10.1128/JVI.78.20.11334-11339.2004 